\title{
Conduta Gerencial do Enfermeiro na Unidade de Terapia Intensiva
}

\author{
SANTOS, José Ribeiro dos
}

SANTOS, José Ribeiro dos. Conduta Gerencial do Enfermeiro na Unidade de Terapia Intensiva. Revista Científica Multidisciplinar Núcleo do Conhecimento. Edição 09. Ano 02, Vol. 01. pp 30-46, Dezembro de 2017. ISSN:2448-0959

\section{Resumo}

Os diferentes níveis e complexidade de assistência prestada aos pacientes requerem do enfermeiro, uma habilidade direcionada para gerenciar recursos físicos, materiais e humano. O enfermeiro se reconhece perto ao cliente, mesmo quando em atividades de cuidado indireto, uma vez que supervisiona o trabalho de sua equipe configura-se como ação de fazer, caracterizada por cuidado direto, cuidado indireto e atos burocráticos. Independente do mapa, o cliente é o objeto de cuidado do enfermeiro sob o princípio "bem cuidado". É dele a responsabilidade de elaborar o planejamento da assistência de enfermagem. O objetivo deste estudo é discutir o processo geral relativo ao gerenciamento de recursos exercido pelo profissional enfermeiro e sua relevância dentro da uma unidade de terapia intensiva. Os resultados apontam que $\mathrm{O}$ enfermeiro está comprometido não só com a administração de recursos humanos, na prestação do cuidado, mas também é de responsabilidade dele prover e providenciar recursos materiais para melhor atender a demanda dos pacientes na sua unidade. Método utilizado Revisão integrativa da literatura. Com isso conclui-se que o enfermeiro é a peça chave no processo de gestão, para uma execução eficiente de gerenciamento, é preciso além de uma visão generalista, ser persuasiva e manipulador de situações adversas.

Palavras-Chave: Gerenciamento de Enfermeiro, UTI, Competência \& Administração.

\section{Introdução}

Os diferentes níveis e complexidade de assistência prestada aos pacientes requerem do enfermeiro, uma habilidade direcionada para gerenciar recursos físicos, materiais e humano. Ao cuidar de pacientes internados em unidades de terapia intensiva (UTIs), a equipe de enfermagem defronta-se constantemente com a binômia vida e morte e, devido às características tecnológicas e científicas, é necessário a 
priorização de procedimentos técnicos de alta complexidade (Martins, et. al. 2009).

O enfermeiro se reconhece perto ao cliente, mesmo quando em atividades de cuidado indireto, uma vez que supervisiona o trabalho de sua equipe. Configura-se como ação de fazer, caracterizada por cuidado direto, cuidado indireto e atos burocráticos. Independente do mapa, o cliente é o objeto de cuidado do enfermeiro sob o princípio "bem cuidado". É dele a responsabilidade de elaborar o planejamento da assistência de enfermagem.

O planejamento procura fornecer informações e dados que permitam uma avaliação das condições gerais do paciente, a orientação das condutas médicas e de Enfermagem, a interação entre os Serviços Médico e de Enfermagem. Embora prevaleça o trabalho fragmentado, é função de o enfermeiro coordenar, realizar e avaliar as atividades da enfermagem, bem como implementar e utilizar o processo de enfermagem para prescrever os cuidados a serem executados. Haag, (2001). Ou seja, deste profissional espera-se que saiba planejar, organizar e avaliar o trabalho da equipe de enfermagem.

O processo de gestão está inserido a transformação de informação em decisões gerais, já a prática, para gerenciar essas decisões estar inserida no processo de efetivação implementada pela instituição. Diante deste contexto o enfermeiro passa a exercer a liderança, estabelecer e definir papeis. Mobilizar recursos, ampliando seus conhecimentos e participando do processo de educação e pesquisa. $\mathrm{O}$ termo "gestão e competência" não são recente, Taylor (1970), já fazia alerta para a necessidade das empresas contratarem trabalhadores eficientes e competentes. Com base nas teorias de Tayloristas, de seleção e treinamento é preciso ressaltar que não basta só treinar, é preciso acompanhar, avaliar e reavaliar esses trabalhadores, entretanto é preciso dar condições para que os resultados esperados sejam eficientes.

A competência é definida como a capacidade decorrente de profundo conhecimento que alguém tem sobre um assunto. Sparrow e Bognanno (1994), ao tratar do mesmo tema, fazem referência a um repertório de atitudes que possibilitam ao profissional adaptar-se rapidamente a um ambiente cada vez menos estável e ter uma orientação para a inovação e a aprendizagem permanente. Segundo esses autores, competências representam atitudes identificadas como relevantes para a obtenção de alto desempenho em um trabalho específico ao longo de uma carreira profissional ou no contexto de uma estratégia corporativa. Existem, ainda, autores que definem competência não apenas como um conjunto de qualificações que o indivíduo detém. Para eles, é necessário também colocar em prática o que se sabe, ou seja, mobilizar e aplicar tais qualificações em um contexto específico.

Estudos realizados por Barreto (2013) revela que na ótica do enfermeiro, a análise dos depoimentos indicou que gerenciar as UTIs significa prestar cuidados ao paciente, administrar a assistência de enfermagem e administrar a equipe de saúde. Que no processo de labor do enfermeiro, a gerência tem papel ímpar, articulando os diversos meios de trabalho da equipe de enfermagem. (gerenciar recursos materiais e humanos).

Com foco no cliente em seu processo de trabalho, ele norteia suas ações a partir da gravidade do quadro clínico da clientela. As unidades de terapia intensiva são importantes recursos para pacientes graves ou pacientes potencialmente graves que necessita de cuidados continuo e especializados.

No Brasil as primeiras UTIs foram instaladas na década de 70, com a finalidade de centralizar pacientes graves recuperáveis, em área hospitalar com recursos humanos, equipamentos e materiais específicos 
direcionados ao cuidado desses pacientes. MIAKO, et al, (2000).

Sabe-se, entretanto que existe uma grande diversidade entre UTIs no que se refere aos recursos estruturais. A Unidade de Terapia Intensiva (UTI) é específica para o tratamento de clientes graves que exige um tratamento intensivo e ininterrupto. Com ênfase na dinâmica e rotinas das UTIs, a cooperação e interação na busca do entendimento e se fazer entender, requer esforços e autoconhecimento entre os colaboradores da equipe em todos os turnos, gerando ou desencadeando mudanças no processo comportamentais e atitudes (Batista, et al, 2007).

A enfermagem tem buscado uma nova configuração, procurando reorganizar-se em um novo modelo assistencial, entretanto continua reproduzindo em seu interior a divisão técnica do trabalho, separando as tarefas gerenciais daquelas direcionadas aos cuidados prestados diretamente ao paciente. Tais dimensões são interdependentes na medida em que, para a exposição de uma habilidade, se presume que o indivíduo conheça princípios e técnicas específicos.

Da mesma forma, a adoção de um comportamento no trabalho exige da pessoa, não raras vezes, a detenção não apenas de conhecimentos, mas, também de habilidades e atitudes apropriadas. Abordagens como essa parecem possuir aceitação mais ampla tanto no ambiente hospitalar como no meio acadêmico, visto que, além do nível individual, o conceito de competência aplica-se também à equipe de trabalho e à organização como um todo.

Constata-se que a competência além de do saber fazer, agrega valores e desempenho superior na realização de tarefas em quais quer situações, o ambiente hospitalar por si só, já causa certa apreensão e quando o ambiente é a unidade de terapia intensiva, causa uma maior expectativa nos pacientes e familiares, exigindo uma maior compreensão e abordagem de comunicação cessível por parte do profissional enfermeiro.

A vida do paciente no hospital depende dos procedimentos diagnóstico-terapêutico e cuidados da equipe de saúde, além de qualidade e disponibilidade como: estrutura física, recursos materiais, humanos e financeiros e quem estão a encargo de gerenciar esses recursos é o Professional enfermeiro (Veiga, 2000).

Ao longo do século XX, a avaliação de desempenho passou das metodologias de controle dos tempos e movimentos para processos que consideram o empregado e seu trabalho como parte de um contexto organizacional e social mais amplo. Diversas empresas têm recorrido à utilização de modelos de gestão e competência.

Esses processos consistem em estabelecer objetivos, metas e prazos a ser alcançados segundo a estratégica adotada pelo enfermeiro responsável pela distribuição das tarefas a ser executadas pela sua equipe. Para que o objetivo proposto pelo enfermeiro seja alcançado, é preciso o engajamento de toda a sua equipe em função assistência individualizada e sistematizada (Coelho, 2004).

No gerenciamento da assistência de enfermagem, cuja meta é o cliente bem cuidado, o enfermeiro se vê próximo ao cliente, ainda que não execute cuidados diretos. Há valorização do planejamento para cuidar de cliente crítico e oferecer condições adequadas de trabalho. (Barreto, 2013).

O planejamento da assistência ao paciente é privativo do profissional enfermeiro, segundo a Resolução do 
COFEN-272-2002, Revogada pela Resolução COFEN nº 358/2009. Esta metodologia é um instrumento privativo do processo de trabalho do enfermeiro, a qual possibilita o desenvolvimento de ações que modificam o estado do processo de vida e de saúde-doença dos indivíduos. Portanto, a Sistematização de Assistência de Enfermagem (SAE), permite que se alcance resultados pelos quais o enfermeiro é responsável.

A implementação da SAE proporciona cuidados individualizados, assim como norteia o processo decisório do enfermeiro nas situações de gerenciamento da equipe de enfermagem ANDRADE, (2005). Sistematizar o cuidado implica em utilizar uma metodologia de trabalho embasada cientificamente. Isto resulta na consolidação da profissão e visibilidade para as ações desempenhadas pelo enfermeiro, bem como oferece subsídios para o desenvolvimento do conhecimento técnico-científico.

O enfermeiro deve ter visão estratégica e atitudes pertinentes ao gerenciar, delegar tarefas aos seus colaboradores, procurando ser justo em suas atitudes, uma vez que o enfermeiro é o responsável pela concepção de gerenciamento do processo de enfermagem, deverá criar mecanismos favoráveis para a execução das tarefas que foram planejadas e implementadas.

A adoção de sistemas de classificação permite o uso de uma linguagem única e padronizada, a qual favorece o processo de comunicação, a compilação de dados para o planejamento da assistência, o desenvolvimento de pesquisas, o processo de ensino-aprendizagem profissional e fundamentalmente confere cientificidade ao cuidado. (Truppel, 2009).

É importante ressaltar que a estrutura física da unidade de terapia intensiva, deve obedecer ás normas técnica e proporcionar um ambiente de segurança para os pacientes e os multiprofissionais, contribuindo para a redução do estresse sobre tudo a carga de trabalho. (Santos, et al, 2009).

O processo do cuidar nas unidades de terapia intensiva requer dos colaboradores não só conhecimento técnico nos procedimentos, mas, também exige o conhecimento técnico-operacional dos profissionais da saúde. No manuseio dos equipamentos, sendo o responsável pelo treinamento e capacitação da equipe, o enfermeiro.

A implementação das estratégicas possibilitam as adequações das unidades viabilizando ideias e dando autonomia aos colaboradores para que possam expressar e criar novos projetos para a melhoria da assistência de enfermagem. (Oliveira, et al., 2009).

No trabalho desenvolvido pelo enfermeiro deve-se compreender que, além do cuidado com os indivíduos hospitalizados, são fundamentais que sejam atendidas as necessidades destes, quer sejam elas biológicas, físicas, psíquicas ou espirituais.

Diante do cuidado altamente especializado e complexo que o enfermeiro desenvolve em uma Unidade de Terapia Intensiva, a sistematização e a organização do seu trabalho e, por conseguinte, do trabalho da equipe de enfermagem, mostram-se imprescindíveis para uma assistência de qualidade, com eficiência e eficácia (Truppel, 2009).

Ao cuidar de pacientes internados em (UTIs), a equipe de enfermagem defrontam-se constantemente situações de vida e morte. Na (UTI), a pesar dos diferentes níveis de complexidade prestados aos clientes, 
cada componente da equipe de enfermagem apresenta distinto perfil profissional. Alguns podem ser recém-formados com pouca experiência na área assistencial, outros já possuem habilidades praticas, mas, não preparados para executar ações de enfermagem complexas, ao passo que outros profissionais já se encontram capacitados para realizar as intervenções prescritas pelo enfermeiro (Balsanelli, 2009).

Estudo realizado por Truppel descreve que a SAE é implementada com todas as suas etapas composto pelos itens históricos, diagnóstico, prescrição e evolução e está embasada na Teoria de Wanda de Aguiar Horta (desenvolvida a partir da Teoria da Motivação Humana de Maslow) e na adoção da taxonomia NANDA para identificar os diagnósticos de enfermagem. Entretanto em seu estudo conclusivo o autor relata que os cuidados prescritos pelos enfermeiros devem ser revistos, por meio de estudos que identifiquem prescrições efetivas e que realmente interfiram no estado clínico do paciente (Truppel, 2009).

As organizações modernas precisam de mecanismos de avaliação de desempenho em diversos níveis: ao nível corporativo, o planejamento, o acompanhamento. A avaliação restringe a missão, visão e ao objetivo a ser Alcançado, tendo a sustentabilidade da organização como Resultado esperado (Oliveira, et al, 2009).

É necessário investimentos e capacitação de todos os profissionais em unidade de terapia intensiva, evitando assim ou mesmo minimizando as ocorrências de iatrogenia no decorrer do atendimento. No âmbito da gestão, a competência foi definida como um saber agir responsável e reconhecido, que implica mobilizar, integrar, transmitir conhecimentos e habilidades que agregam valores econômicos à organização e valor.

De acordo com Rika et. al. (2010), do social ao individuo. Surge então a necessidade de que a construção de competências profissionais ocorra no contexto organizacional, na capacitação continua e na gestão de recursos sejam eles humanos ou materiais.

Já de acordo com Fernandes et. al. (2003), a busca do cliente por produtos e serviços de qualidade, o crescente avanço tecnológico em diversas áreas, mudanças nos processos de trabalho, problemas financeiros, entre outros, são fatores que estão exigindo das empresas públicas e privadas adaptação rápida e constante às mudanças e instabilidades dos tempos atuais. Nesse contexto, observa-se que a conduta do gerente deve ser modificada perante as demandas contemporâneas.

A organização do trabalho baseada na formação de equipes, "certamente tem sido a forma mais democrática, produtiva e humanizada de se efetuar o trabalho em saúde". Spagnol, (2002). O treinamento, assim como o desenvolvimento da equipe de enfermagem, justifica-se pela promoção de capacitação técnica específica, da aquisição de novos conceitos, atitudes e da visão crítica dos problemas contemporâneos, responsabilidades sociais e cooperação dentro e fora do ambiente de trabalho. A enfermagem avança continuamente incorporando novas tecnologias que devem ser revertidas na qualificação do cuidado prestado. Apesar de prevalecer na enfermagem uma raiz histórica de autoritarismo, centralização das decisões e impessoalidade nas relações, ainda presentes nos dias atuais, percebe-se que a introdução de novas abordagens gerenciais, devido às intensas transformações que estão ocorrendo na sociedade, está impulsionando os gerentes a buscarem novas alternativas para organizar o trabalho.

Segundo a autora Fernandes, (2003), essas novas abordagens trazem no seu bojo conceitos de 
flexibilidade, redução da hierarquia, trabalho em equipe e descentralização das decisões, visando à satisfação dos clientes e trabalhadores, bem como a produtividade e a responsabilidade compartilhada, que devem ser vislumbradas pelos gerentes do futuro.

No que tange à prática profissional do enfermeiro, percebe-se e destaca-se o valor da liderança, pois é através dela que este profissional garante uma boa gerência e melhoria da qualidade da assistência de enfermagem (Santos, 2010). É notório que em muitas das instituições de saúde, ainda rege os preceitos das teorias clássicas, centralizando o poder de decisão em um grupo seleto de pessoas, que na maioria das vezes estão dispersas dos reais problemas que eventualmente possam ocorrer na base.

Assim, de acordo com Santos (2010), paradoxalmente, nos modelos gerenciais mais contemporâneos, pautados em estruturas flexíveis, descentralizadas e ligados à responsabilização dos envolvidos, deve-se pensar menos intensivamente em comunicação vertical e, prioritariamente, em comunicação horizontal ou Lateral, incentivando tal processo tanto interunidades como intraunidade.

Ao longo desta trajetória histórica, com a implementação da SAE, com as mudanças estruturais além da competência assistencial e ensino, foram requeridos competência de gestão e pesquisa exigindo o desenvolvimento de competências profissionais que agregam valores à instituição e aos indivíduos que contribuíram para os resultados organizacionais (Fleury, 2004).

Para atingir o sucesso organizacional nas instituições, o enfermeiro deve ser o elo da cadeia comunicativa, uma vez que está constantemente em contato com a equipe multiprofissional. Além disso, a insuficiência do processo de comunicação é responsável pelo desencadeamento de fatores geradores de insatisfação nas instituições de saúde. Na gestão de pessoas por competências, algumas premissas essências, como foco no desenvolvimento das pessoas, no processo, na conciliação dos interesses da empresa e do profissional. Um modelo integrado e estratégico, em vez de constituído por partes desarticuladas entre si ( Dutra, 2002).

Autores cita o processo comunicativo como um dos pontos necessários para se alcançar o êxito gerencial em instituições de saúde, salientando as várias contribuições que a comunicação clara e adequada é capaz de propiciar às equipes. Quando o modelo gerencial da instituição é pautado na Abordagem Clássica da Administração a comunicação verticalizada, truncada e, portanto, ineficiente. Já quando se adota o estilo contemporâneo de gestão, busca-se a intensificação da comunicação em todos os níveis, ou seja, vertical e horizontalmente ( Santos, 2010).

Neste contexto, busca-se enfatizar o cuidado/assistência como eixo principal do trabalho em enfermagem, explorando o campo de atividade dos enfermeiros responsáveis por unidade de terapia intensiva, tentando identificar a importância/relevância que estes atribuem para a prestação do cuidado.

Frente ao que foi exposto se faz necessário a indagação: qual seria a relevância do profissional enfermeiro dentro de uma unidade de terapia intensiva? Quais seriam as competências exigidas para esse profissional dentro do processo do cuidado? E até que ponto o enfermeiro estaria preparado para exercer a função de gestão competente? Abordaremos aqui algumas questões pertinentes atribuídas ao profissional enfermeiro nas unidades de terapia intensiva.

O objetivo é discutir o processo geral relativo ao gerenciamento de recursos exercido pelo profissional 
enfermeiro e sua relevância dentro da uma unidade de terapia intensiva.

\section{MATERIAIS E MÉTODOS}

Revisão integrativa da literatura. Os artigos foram pesquisados no banco de dados on-line. Toda revisão literária passou por um processo sistemático de análise e foram selecionados os artigos inerentes ao assunto abordado, após o levantamento das citações e posterior fichamento, cuja descrição atendeu os objetivos do estudo proposto. Foram encontrados 45 artigos científicos, compreendendo o período de 2000 a 2015, foram selecionados temas pertinentes ao assunto abordado. O levantamento dos dados realizado em fontes como: (Lilacs) Literatura-Americana do Caribe, (SciELO) - Scientific Electronic Library Online, (BIREME) Biblioteca Virtual de Medicina; Medical Literature Analysis and Retrieval Systems Online (MEDLINE) e dados das seguintes entidades: (COREN-SP) Conselho Regional de Enfermagem DE São Paulo. Para a pesquisa deste trabalho foram usados os descritores: UTI, Enfermagem, Gerenciamento \& Competência. Ao utilizar os descritos citados, foram encontrados 45 artigos que estavam disponíveis na íntegra. Inicialmente procedeu-se a checagem dos títulos, autores e resumos, com o objetivo de separar as publicações repetidas, a seguir foram esmiuçadas todas as publicações encontradas e selecionados os artigos inerente ao assunto abordado. Foram descartados 22 artigos por não se adequarem ao objetivo e usado às demais publicações para compor esse estudo.

\section{RESULTADOS E DISCUSSÕES}

Tabela 1 - Caracterização dos artigos por ano de sua publicação. São Paulo, 2016.

\begin{tabular}{|c|c|c|}
\hline Ano & F & \% \\
\hline $\mathbf{2 0 1 5}$ & 00 & 0,00 \\
\hline $\mathbf{2 0 1 4}$ & 00 & 0,00 \\
\hline $\mathbf{2 0 1 3}$ & 01 & 4,34 \\
\hline $\mathbf{2 0 1 0}$ & 00 & 0,00 \\
\hline $\mathbf{2 0 0 9}$ & 01 & 4,34 \\
\hline $\mathbf{2 0 0 8}$ & 02 & 8,69 \\
\hline $\mathbf{2 0 0 7}$ & 06 & 26,08 \\
\hline $\mathbf{2 0 0 5}$ & 02 & 8,69 \\
\hline $\mathbf{2 0 0 4}$ & 01 & 4,34 \\
\hline $\mathbf{2 0 0 3}$ & 00 & 0,00 \\
\hline $\mathbf{2 0 0 2}$ & 01 & 4,34 \\
\hline $\mathbf{2 0 0 1}$ & 02 & 8,69 \\
\hline $\mathbf{2 0 0 0}$ & 01 & 4,34 \\
\hline & 02 & 8,69 \\
\hline & 02 & 8,69 \\
\hline
\end{tabular}




\section{Total}

Tabela 1- Pode observar conforme mostra a tabela acima que houve uma instabilidade entre os anos de publicações de 2000 a 2009, havendo um aumento importante de publicações no ano de 2009, num total de 06 artigos publicados representando um percentual de (26\%).

É possível observar que nos anos de 2006, 2012, 2014 e 2015, não foram encontrado artigos que se adequassem a temática em questão. Já os anos de 2000, 2001, 2002, 2004, 2008 e 2010, foram encontrados duas publicações em cada ano, juntas eles formam um total de 12 publicações, representando um percentual de $(52,17 \%)$.

Também é possível observar que os anos de menor número de publicação foram os anos de 2003, 2005, 2007, 2011 e 2013. Foi encontrado apenas um artigo que se adequasse ao abjetivo do estudo, juntos somam um total de 5 artigos, representando um percentual de $(21,73 \%)$.

Tabela 2 - Caracterização da amostra por temas gerenciais abordados na literatura, São Paulo, 2016.

\begin{tabular}{|c|c|c|}
\hline Temas Gerenciais & $\mathbf{n}$ & \% \\
\hline Gerenciamento & 06 & 26,08 \\
\hline Competência & 04 & 17,39 \\
\hline Liderança & 03 & 13,04 \\
\hline Carga de Trabalho & O3 & 13,04 \\
\hline SAE & 03 & 13,04 \\
\hline Comunicação & 01 & 4,34 \\
\hline Educação & 01 & 4,34 \\
\hline Administração & 02 & 8,69 \\
\hline Total & $\mathbf{2 3}$ & $\mathbf{1 0 0}$ \\
\hline
\end{tabular}

Fonte: Dados elaborados pelo autor

Devido o grande número de artigos encontrados relacionado ao gerenciamento, foram separados os artigos por temas: gerenciamento, competência, liderança, administração, carga de trabalho, comunicação, Sistematização da Assistência de Enfermagem (SAE) e educação.

Verifica-se que, a temática com maior número de publicações foram gestão e competência, gestão foram encontrados 6 artigos $(n=26,08 \%)$ e competência 4 artigos $(n=17,39 \%)$. É possível observar que os temas: liderança, carga de trabalho e SAE, foi encontrados 3 artigos por temas ( $\mathrm{n}=13,04 \%$ ), somam juntos 9 artigos representando um percentual de $(39,13 \%)$.

Já 0 tema referente a administração, foram encontrados 2 artigos $(n=8,69)$. De acordo com a tabela, os 
temas educação e comunicação foram encontrados o menor número de publicações, apenas 1 artigo cada $(n=4,34 \%)$, juntos somam um percentual de $(8,68 \%)$.

Como gerenciamento se depara com administração, foram revistos também as teorias de Taylor um dos pilares das teorias clássicas, que norteiam as tarefas desenvolvidas pelos profissionais da enfermagem nas instituições públicas e privadas de saúde de todo o país.

Nesse contexto, as UTIs tornaram-se unidades onde se concentram recursos humanos e tecnológicos altamente especializados que proporcionam assistência considerada como das mais complexas, sofisticadas e onerosas do sistema de atendimento à saúde. Dentre os vários métodos científicos específicos das ciências da Enfermagem, o Processo de Enfermagem, a (SAE) se adapta aos objetivos da assistência, indo ao encontro da dinâmica das ações sistematizadas e inter-relacionadas, visando à assistência ao ser humano.

O enfermeiro esta comprometido não só com a administração de recursos humanos, mas tambem é de responsabilidade dele prover e providenciar recursos materiais para melhor atender a demanda dos pacientes na sua unidade, o melhor gestor de custos é quem conhece profundamente as operações e não quem reúne apenas os conceitos relacionados à contabilização dos custos (Santos, 2009).

Outro fator importante dentro da UTI é a administração do tempo. Alocar tempo para o planejamento e estabelecer prioridades; Completar a tarefa de mais alta prioridade sempre que possível e terminar uma tarefa antes de iniciar outra; Estabelecer novas prioridades com base nas tarefas remanescentes e nas novas informações que tenham sido recebidas. Tudo isso leva tempo, requer competência e capacidade de pensar, analisar dados, visualizar alternativas e tomar decisões.

Para que não ocorra uma discrepância entre as atividades desenvolvidas pelos enfermeiros e as delegadas aos outros funcionários, é preciso que se tenha um planejamento adequado das atividades, pois os serviços de enfermagem dentro de uma UTI são complexos, exigindo uma resposta imediata, ou seja, envolve estudo e uso de fatos e princípios, exigindo dessa forma conhecimento, imaginação, raciocínio, habilidade e técnica de pesquisa (Gonçalves, 2001).

Com o passar do tempo, a enfermagem em terapia intensiva incorporou alguns papéis, as ações assistenciais praticadas pelos enfermeiros junto aos pacientes o mais expressivo deles é consequentemente a crescente participação em tomada de decisão (Viana, 2011).

Houve a necessidade do enfermeiro além de lidar com sua equipe, desenvolver mecanismos e habilidades para gerenciar as tarefas a qual lhe são atribuídas. Hoje é possível dimensionar as ações do enfermeiro e suas equipes de trabalho nas UTIs atendimento direcionado, com atendimento com maior rigor as necessidades dos pacientes.

Tabela 3 - Distribuições dos artigos conforme as respectivas revistas. São Paulo, 2016

\begin{tabular}{|c|c|c|}
\hline REVISTAS & n & \% \\
\hline $\begin{array}{c}\text { Rev. da Escola de Enfermagem } \\
\text { da USP }\end{array}$ & 7 & 30,43 \\
\hline
\end{tabular}




\begin{tabular}{|c|c|c|}
\hline $\begin{array}{c}\text { Rev. Latino-Americana de } \\
\text { Enfergaem }\end{array}$ & 6 & 26,08 \\
\hline \begin{tabular}{c|c|} 
Rev. Brasileira de Enfermagem. \\
Revista de Administração de \\
Empresas.
\end{tabular} & 4 & 17,39 \\
\hline Editora Artmed & 1 & 4,34 \\
\hline Rev. Brasileira de Terapia & 1 & 4,34 \\
\hline Intensiva. & 1 & 4,34 \\
\hline U.F. Fluminense & 2 & 4,34 \\
\hline Rev. Gaúcha de Enfermagem. & $\mathbf{2 3}$ & 8,69 \\
\hline Total & & $\mathbf{1 0 0}$ \\
\hline
\end{tabular}

Fonte: Dados elaborados pelo autor

De acordo com os dados apresentados na tabela acima, notamos um aumento acentuado de publicações na Revista da Escola de Enfermagem da USP num total de 7 publicações. Representando um percentual de $(30,43 \%)$.

Em seguida com 6 publicações, a Revista Latino-America de Enfermagem, com (26,08\%), seguida pela Revista Brasileira de Enfermagem com 4 artigos (17,39\%).

Na Revista Gaúcha de Enfermagem foram encontradas 2 publicações (8,69\%). E nas revistas: Editora Armed; Revista de Administração de Empresas; Revista Brasileira de terapia Intensiva; Revista da Universidade Federal Fluminense foi encontrada uma publicação em cada revista. (4,34\%). Juntas elas somam um total de 4 artigos, representando um percentual de $(17,36 \%)$.

A carga de trabalho é um fator que preocupa os gestores das unidades de terapia intensiva, sabe-se que os excessos de carga de trabalho e os procedimentos cada vez mais complexos, exigem uma atenção maior dos colaboradores. Pesquisas apontam que funcionários cansados com sobre carga de tarefas tem maior possibilidade de cometer erros do que aqueles que estão descansados. A segurança do paciente no decorrer da internação hospitalar tem merecido atenção crescente na qualidade e o mínimo de risco possível pra o paciente com a implementação de protocolos de risco de quedas.

No Brasil, por meio das Diretrizes Curriculares Nacionais (DCNs,) para os Cursos de Graduação em Enfermagem em vigor, tenta-se orientar para competências gerais a serem alcançadas pelo futuro enfermeiro, como a atenção à saúde, a tomada de decisão, a comunicação, liderança, administração e gerenciamento e educação permanente.

Pensando nisso, estudos vêm sendo realizados sobre a competência profissional do enfermeiro e destacase pesquisa que investigou competências gerenciais do enfermeiro, relacionadas às expectativas da instituição formadora e o mercado de trabalho. Na literatura mundial, constata-se a importância da atuação do enfermeiro na UTIs, no entanto, observa-se, no dia a dia, dicotomia entre o que se aprende 
durante a formação e o que é realizado na prática. A prática da enfermagem, baseada em evidências científicas, a divulgação de pesquisas relacionadas ao cuidado de enfermagem e a capacidade de padronizar o cuidado, de supervisionar o trabalho da equipe de enfermagem.

\section{CONCLUSÃO}

Os dados do presente estudo, conclui-se que o enfermeiro é a peça chave no processo de gestão, para uma execução eficiente de gerenciamento, é preciso além de uma visão generalista, ser persuasivo e manipulador de situações adversas.

O trabalho do enfermeiro em uma Unidade de Terapia Intensiva (UTI) é caracterizado por atividades assistenciais e gerenciais complexas que exigem competência técnica e científica, cuja tomada de decisões e adoção de condutas seguras estão diretamente relacionadas à vida e à morte das pessoas. Nesse contexto, é de suma importância identificar as competências desses profissionais ao desenvolver a assistência de enfermagem de alta complexidade, como é o caso da UTI.

A luz das incertezas do setor, se da na melhoria continua, expansão dos conhecimentos, manuseio dos equipamentos e mão de obra qualificada. Faz-se necessário salientar que para um gerenciamento eficiente, deverá haver condições favoráveis para sua aplicabilidade.

O enfermeiro assume a função de supervisão da equipe de enfermagem, independentemente do cargo, sendo a educação uma de suas atividades de maior relevância junto a seu pessoal. Supervisão como sendo um processo educativo que visa à motivação e à orientação para sua equipe. As atribuições e competências do enfermeiro é um profissional que acumula funções e responsabilidades, sendo que, o pleno desempenho de suas tarefas, pode levar a recuperação melhor e mais rápida dos pacientes.

O exercício profissional do enfermeiro pauta-se na relação interpessoal enfermeiro-paciente- famíliaequipe. A utilização da tecnologia variavelmente parece tangenciar esta relação, trazendo algumas dúvidas, inseguranças e receios dos profissionais em se apropriar da tecnologia, como se fosse interferir negativamente na qualidade da assistência. Há evidências, que a incorporação tecnológica em nada fragmenta o cuidado, uma vez que a utilização destes recursos pode aperfeiçoar a qualidade da assistência. O enfermeiro tem a possibilidade de apropriar-se disso para o desenvolvimento de estratégias de educação permanente da equipe de enfermagem e correlacionar com indicadores gerenciais e assistenciais da unidade.

\section{REFERÊNCIAS}

BALSANELLI, Alexandre Pazetto; Cunha, Isabel Cristina Kowal Olm; Whitaker, Iveth Yamaguchi. Estilos de lideranças de enfermeiros em unidade de terapia intensiva: associação com o perfil com o perfil pessoal , profissional e carga ded trabalho. Revista Latina-Americana de Enfermagem. 17 (1). Jan./fev.2009.

BARRETO VPM, Tonini T, Aguiar BGC. Nursing care management of clients in intensive care: content analysis. Online braz j nurs [Internet]. 2013 Oct [cited year mouth day]; 12 Suppl: 578-80. Available from: http://www.objnursing.uff.br/index.php/ nursing/article/view/4076. 
BRAANDÃO, Hugo Pena; Guimarães, Tomás de Aquino. Gestão de competência e gestão dee desempenho : tecnologias distintas ou instrumentos do mesmmo consumo? Revista de Administração dde Empresas REA. Rio ded Janeiro. Mar./2001

COELHO, Celso Dias. Para além dadas óptticas restritivas das avaliações: uma visão da terapia intensiva. Rio de Janeiro s.n; p. 182, 2004.

DUTRA, J.S. Modelo de gestão de pessoas: modelos, procecsos, tendências e perspectivas. Ed. Atlas. P.41-60 mar./2002.São Paulo.

FERNANDE, Marcia Simoni; Spagnol, Carla Aparecida; Trevizan, Maria Auxiliadora; Hayashida, Miyeko. A conduta gerencial da enfermeira: um estudo fundamentado nas teorias gerais da administração. Revista Latino-Americana de Enfermagem vol 11 n 2 Ribeirão Prreto. Mar./apr. 2003

FIGUEIREDO, M.R.B; Costa, S.C; Schaurichd, D. Compreensão da equipe ded enfermagem em unnidade de terapia intensiva adulto. Porto Alegrre, vol. 13 p. 575-80, 2009.

FLEURY, A. MTL. Estratégicacs empresariais e formação de competência: um quebra-cabeça

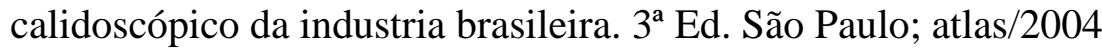

GONÇALVESS, L.A; Padilha, K.G. Fatores associados à carga dde trabalho em unidaded de terapia intensiva. Revista da Escola de Enfermagem USP 2001.

MADUREIRA, Cátia Romanno; Veiga, Kátia; Sant'ana, Ana Flávia Mota.. Gerenciamento de ttecnologia em terapia intensiva. Revista Latina-Americana ded Enfermagem. Vol $8 n^{\circ} 6$ Ribeirão PretoSão Paulo dez/2000.

MARTINS, Juliana Trevissan; Robazzi, Maria Lúcia do Caarmo Cruz; Marziela, Maria Helena Palocci; Haddad, Maria do Carmo Lourenço. Gerenciamento de enfermagem de unidade de terapia intensiva. Revvista Gaúchaa de Enfermagem. 30 (1): 113-119. Março/2009.

MIAKO, Kimura; Kaizumi, Maria Sumier; Martins, Luciana Monteiro Menddes. Caracterização das unidades de terapia intensiva do município de são Paulo. Revista daa Escola dde Enfermagem da USP. Vol.331 n², São Paulo/2000.

NURSING ACTIVITIES SCORE (NAS) como instrumento para medir carga de trabalho de enfermagem em UTI adulto. Regina Maria Yatsue Conishi1, Raquel Rapone Gaidzinski. Rev Esc Enferm USP 2007; 41(3):346-54. www.ee.usp.br/reeusp/346.

OLIVEIRA, José Carlos de; Prado, Claúdia; Peres, Heloísa Helena Ciqueto.; Fernandes, Maria de Fátima; Leite, Maria Maadalena Januário. Grau de competência gerencial em enfermagem na perspectiva de graduados de uma universidade privada. Revista da Escola de Enfermagem da USP vol. 43 n $^{\circ}$ 2. São Paulo ddez./2009

OLIVEIRA, Naiara Cristina de; Chaaves, Luciele Dias Pedreschi. Gerenciamento derecursos materiais: o papel do enfermeiro de uma unidade de terapia intensiva. Revista Rene; 10 (4): 19-27. Dez/2009. 
RIKA, Miyahara Kobayshi. Leite, Maria Madalena Januário. Desenvolvendo competência profissionais dos enfermeiros em serviços. Revista Brasileira de Enfermagem v. 63 n² Brasília março/2010.

SANTOS, José Luis Guededs dos;Galert, Stela Regina; Limma, Maria Alice Dias da Silva. Revisão sistemática sobrer a dimensão gerencial no trabalho do enfermeiro no âmbito hospitalar. Revista Gaúcha de Enfermagem. 30 (3); 525-552 set./2009.

SANTOS, Maria Cláudia dos; Bernardes, Andrea. Comunicação da equipe de enfermagem e a relação com a gerência nas instituições de saúde. Rev. Gaúcha Enferm. (Online) vol.31 no.2 Porto Alegre June 2010.

SILVA, S.C.; Padilha, K.G. Paradacrdio-respiratória na unidaded de terapia intensiva: a analise das ocorrências iatrogênnicaas durante o atendimento. Revista da Escola de Enfermagem da USP. VOL. 34 $\mathrm{N}^{\circ}$ 4, São Paulo/2000.

SOUZA, S.R.O.S; Silva, C.A; Mello, U.M; Ferreia, C..N. Aplicabilidadde de idicadores de qualidade: objetivo em terapia intensiva. Revista Brsileira de Enfermagem. 2008.

SPAGNOL CA, Ferraz CA.Tendências e perspectivas da administração em enfermagem: um estudo na Santa Casa de Belo Horizonte-MG. Rev Latino-am Enfermagem 2002 janeiro/fevereiro.

TRUPPEL, Thiago Christel; Meier, Marineli Joaquim; Calixto, ; Riciana do Carmo; Peruzzo, Simone Aparecida; Crozeta, ; Karla. Sistematização da Assistência de Enfermagem em Unidade de Terapia Intensiva. Revista Brasileira de Enfermagem vol. 62 n² 2 Brasíçia mar./apr. 2009.

[1] Mestrando em Ciências da Educação pela Universidad Politécnica y Artística Del Paraguay. Especialista em Urgência e Emergência com ênfase em APH, pela Faculdade de Ciências Médicas da Santa Casa de São Paulo, Especialista em Docência do Ensino Médio, Técnico e Superior pela Faculdade Associada Brasil. Bacharel em Enfermagem pela Universidade Paulista- UNIP. Licenciado em Biologia pelo Centro Universitário Claretiano. Atualmente é palestrante, professor dos cursos da Pós-Graduação da Faculdade Associada Brasil nas áreas de Educação e Saúde, professor técnico/pedagógico da Escola G12 Educacional CEENPRO

\section{PUBLIQUE SEU ARTIGO CIENTÍFICO EM:}

https://www.nucleodoconhecimento.com.br/enviar-artigo-cientifico-para-submissao 